\section{Le complexe KLHL12/Culline-3 forme une nouvelle ubiquitine ligase $\varepsilon 3$ pour la régulation de la voie Wnt}

Stéphane Angers

\author{
Leslie Dan Faculty of Pharmacy, \\ University of Toronto, Toronto, \\ Ontario M5S 3M2, Canada. \\ stephane.angers@utoronto.ca
}

sant l'activation de Dsh par les récepteurs Frizzled de même que la modulation des différents effecteurs de Dsh sont toutefois encore mal compris.

Nous avons optimisé la méthodologie TAPTAG (tandem affinity purification) facilitant la séparation de complexes protéiques à partir de cellules de mammifères. En utilisant cette approche, nous avons isolé les complexes protéiques pour Dsh-2 et

(adenomatous polyposis coli), Axine et $\beta$-caténine, qui régulent la voie de transduction du signal en réponse aux Wnt, ont été associées à certains cancers chez l'homme. Il est donc important de comprendre les voies de signalisation de Wnt.

Les Wnt activent au moins trois voies de signalisation intracellulaires distinctes qui ont été relativement bien déchiffrées à la suite de cribles génétiques effectués chez la drosophile et chez le nématode C. elegans. Parmi ces trois voies, la voie de signalisation Wnt dépendante de l'activité de la protéine $\beta$-caténine est la mieux caractérisée. La stabilité post-traductionnelle de la $\beta$-caténine, reposant sur le contrôle de son ubiquitinylation et sur son acheminement vers le protéasome, est le mécanisme de régulation le plus important de cette voie (Figure 1A). Après la reconnaissance du ligand Wnt par le récepteur membranaire Frizzled et par le co-récepteur low density related polypeptide 5/6 (LRP5/6), la protéine cytoplasmique Dishevelled (Dsh) est activée par un mécanisme encore mal défini qui conduit à l'inhibition de l'ubiquitinylation de la $\beta$-caténine et donc à sa stabilisation et à son

accumulation dans le cytoplasme. Par la suite, la $\beta$-caténine est transportée dans le noyau où elle modifie l'expression de gènes cibles (Figure 1B). Les deux autres voies Wnt sont indépendantes de l'activité de la $\beta$-caténine et régulent la polarité [2], l'adhérence ou la migration cellulaire. Les trois voies de signalisation mettent en jeu la protéine cytoplasmique Dsh. Les mécanismes moléculaires régis-

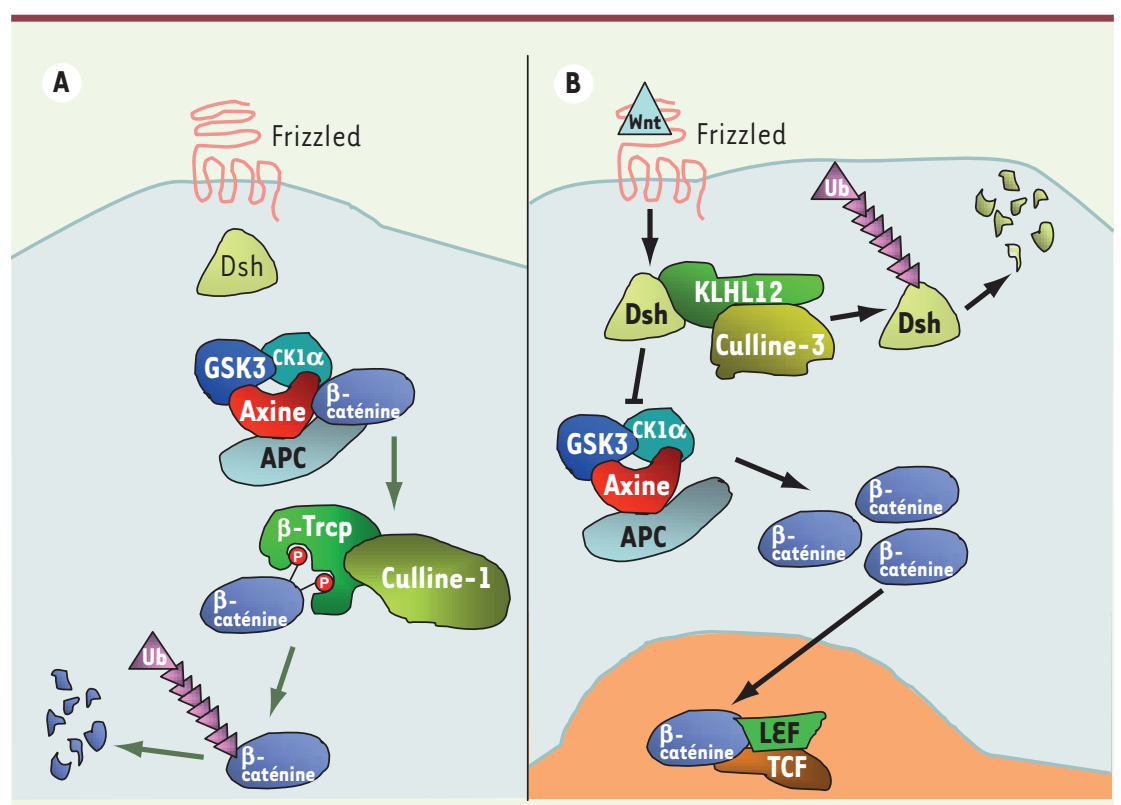

Figure 1. Régulation de la voie Wnt par deux $\varepsilon 3$ ubiquitines ligases. A. À l'état de base, le complexe de destruction (APC, Axine, GSK3, CKla) catalyse la phosphorylation de la $\beta$-caténine. Phosphorylée, la $\beta$-caténine devient un substrat de haute affinité pour une $\varepsilon 3$ ubiquitine ligase de type SCF (Skpl, F-Box ( $\beta$-Trcp), Culline-1) qui entraîne sa dégradation. B. À la suite de la liaison de Wnt à son récepteur Frizzled, la protéine cytoplasmique Dishevelled (Dsh) est activée. Dishevelled inhibe le complexe de destruction, ce qui entraîne l'accumulation de la $\beta$-caténine. La $\beta$-caténine est transportée dans le noyau où, en se liant aux protéines Lef (lymphoid enhancer factor-1) et Tcf ( $T$-cell factor), elle pourra modifier l'expression de gènes. La $\varepsilon 3$ ubiquitine ligase, formée de la Culline-3 et de l'adaptateur KLHL12, recrute Dishevelled pour l'ubiquitinylation et la dégradation, formant ainsi une nouvelle boucle de régulation négative pour la voie de signalisation. 
Dsh-3, deux des trois gènes codant pour Dsh chez les vertébrés, et déterminé l'identité des différents membres des complexes à l'aide de la spectroscopie de masse [3]. Confirmant l'efficacité de notre approche protéomique, nous avons identifié lors de nos analyses plusieurs protéines dont l'interaction avec Dsh était déjà connue. Parmi les 16 protéines que nous avons mises en évidence, se trouvent les sousunités $\beta$ et $\gamma$ des protéines $G$ hétérotrimériques dont le rôle dans la signalisation en réponse aux Wnt demeure toujours controversé. L'étude par spectroscopie de masse des complexes formés par Dsh-2 et Dsh-3 montre également une présence prépondérante de la protéine KLHL12 [3]. Cette protéine contient un domaine BTB (broad complex, tramtrack et «bric à brac») en amino-terminal. Récemment, il a été établi que les protéines contenant un domaine BTB peuvent servir d'adaptateurs spécifiques au sein des ubiquitine ligases $\varepsilon 3$ de la famille Culline-3 pour le recrutement de substrats vers la machinerie d'ubiquitinylation [4]. Étant donné que la Culline- 3 se trouve aussi dans la liste des protéines identifiées lors de l'analyse des complexes Dsh-2 et Dsh3 , nous avons testé I'hypothèse selon laquelle Dsh pouvait être régulée par cette ubiquitine ligase par l'entremise de la protéine adaptrice KLHL12. Des approches de gain de fonction et de perte de fonction en utilisant les embryons de xénope et du poisson zèbre comme modèles expérimentaux, ainsi que des essais fonctionnels en culture cellulaire ont permis de mettre en évidence que KLHL12 agit comme un nouveau régulateur négatif des voies de signalisation Wnt. Les résultats orientent vers la formation dépendante de l'activation de la voie Wnt d'un complexe formé de Culline3, KLHL12 et du substrat Dsh, requis pour I'ubiquitinylation et la dégradation de Dsh par le protéasome.

La régulation de la voie Wnt par une ubiquitine ligase $\varepsilon 3$ de la famille Culline-1, qui emploie une famille de protéines contenant un domaine F-Box pour la reconnaissance des substrats, a bien été caractérisée pour régir la dégradation de la $\beta$-caténine [5]. Le fait qu'il ait été démontré que deux ubiquitine ligases $\varepsilon 3$ recourant à des modes différents pour la reconnaissance des substrats (Culline-1/F-Box ${ }^{\beta-T r c p} / \beta$-caténine, ainsi

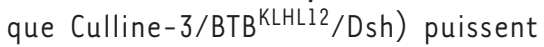
agir au sein d'une même voie de signalisation illustre bien la redondance des mécanismes de régulation cellulaire qui assurent la régulation fine et l'intégrité du signal. Il a été suggéré que d'autres protéines, telles Naked, Inversin et PP2a (protein phosphatase type 2A), pourraient moduler la stabilité protéique de Dsh. Des recherches supplémentaires sont maintenant nécessaires pour comprendre l'interdépendance entre ces différents facteurs et la séquence d'événements qui conduit à la régulation de l'activité de Dsh et de la voie Wnt. Aussi, contrairement aux $\varepsilon 3$ de la famille
Culline-1 où la phosphorylation du substrat est généralement nécessaire pour leur recrutement, la famille Culline-3 ne semble pas, elle, dépendre de la modification post-traductionnelle du substrat pour le recrutement vers la machinerie d'ubiquitinylation. Les mécanismes moléculaires permettant le recrutement de Dsh vers KLHL12 et Cullin-3, et donc nécessaires à sa dégradation, comme à sa régulation, restent à être éclaircis. Considérant qu'une mauvaise régulation de la voie Wnt a été associée à plusieurs formes de cancers (côlon, sein et foie) chez l'humain, la clarification sur le plan moléculaire de nouveaux mécanismes de régulation de cette importante voie de signalisation pourrait mener à comprendre l'étiologie de ces pathologies et offrir de nouvelles stratégies pour leur traitement. $\diamond$

KLHL12/Cullin-3 is a new $\varepsilon 3$ ubiquitin ligase negatively regulating

the Wnt pathway

\section{RÉFÉRENCES}

1. Wodarz A, Nusse R. Mechanisms of Wnt signaling in development. Annu Rev Cell Dev Biol 1998 ; 14 : 59-88.

2. Schweisguth $F$. Bases génétiques de la polarité planaire. Med Sci (Paris) 2004 ; $20: 424-30$.

3. Angers S, Thorpe CJ, Biechele TL, et al. The KLHL12Cullin-3 ubiquitin ligase negatively regulates the Wntbeta-catenin pathway by targeting Dishevelled for degradation. Nat Cell Biol $2006 ; 8$ : 348-57.

4. Van den Heuvel S. Protein degradation: CUL-3 and BTB--partners in proteolysis. Curr Biol 2004 ; 14 : R59-61.

5. Latres $\varepsilon$, Chiaur DS, Pagano M. The human F box protein beta-Trcp associates with the Cull/Skpl complex and regulates the stability of beta-catenin. Oncogene $1999 ; 18: 849-54$

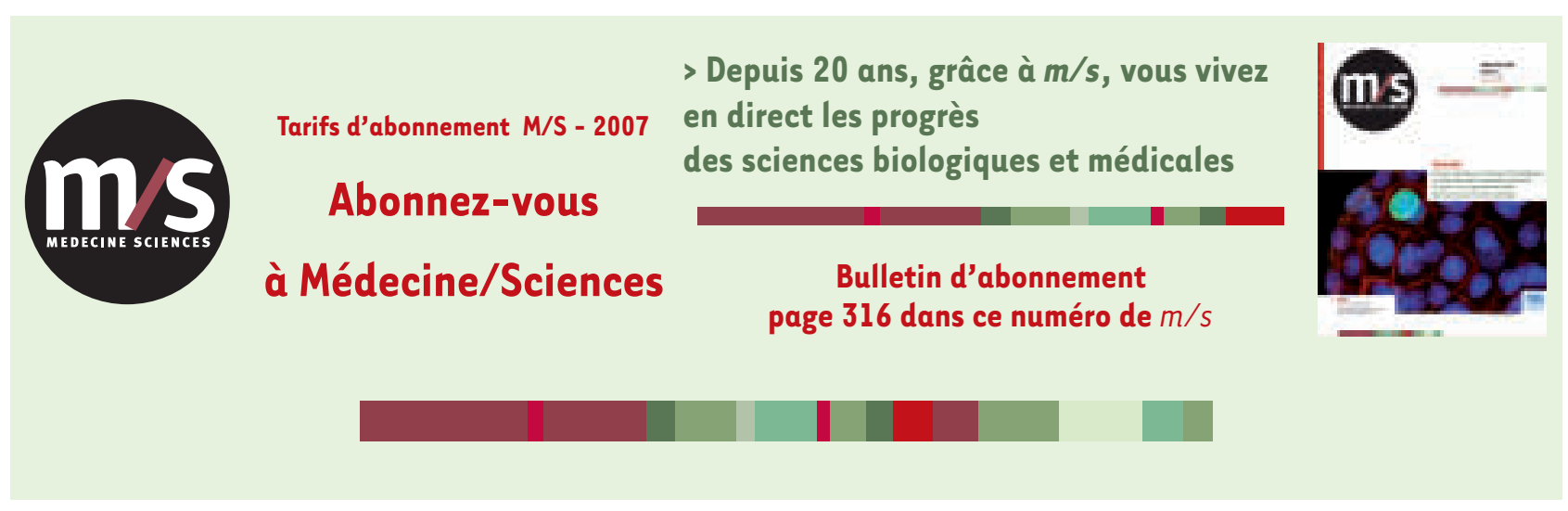

